\title{
Postnatal LPS Challenge Impacts Escape Learning and Expression of Plasticity Factors Mmp9 and Timp1 in Rats: Effects of Repeated Training
}

\author{
Alexander Trofimov ${ }^{1,2,3} \cdot$ Tatyana Strekalova $^{1,4} \cdot$ Niall Mortimer $^{1} \cdot$ Olga Zubareva $^{2,5}$. $^{2}$ \\ Alexander Schwarz ${ }^{2}$ Evgeniy Svirin ${ }^{6}$ - Aleksei Umriukhin ${ }^{6,7} \cdot$ Andrei Svistunov ${ }^{6}$ • \\ Klaus-Peter Lesch ${ }^{1}$ • Victor Klimenko ${ }^{2}$
}

Received: 16 November 2016/Revised: 1 March 2017 / Accepted: 3 March 2017 /Published online: 18 April 2017

(C) The Author(s) 2017. This article is an open access publication

\begin{abstract}
Bacterial intoxication associated with inflammatory conditions during development can impair brain functions, in particular evolutionarily novel forms of memory, such as explicit learning. Little is known about the dangers of earlylife inflammation on more basic forms of learning, for example, the acquisition of motor escape abilities, which are generally better preserved under pathological conditions. To address this limitation in knowledge, an inflammatory response was elicited in Wistar pups by lipopolysaccharide (LPS) injections $(25 \mu \mathrm{g} / \mathrm{kg})$ on postnatal days P15, P18 and P21. The acquisition of escape behaviour was tested from P77 by active avoidance footshock model and water maze. Open-field behaviour and blood corticosterone levels were also measured. Rat brain tissue was collected from pups $2 \mathrm{~h}$ post-injection and from adult rats which either underwent escape training on P77-P81 or remained untrained. mRNA levels of
\end{abstract}

Alexander Trofimov and Tatyana Strekalova equally contributed to this work.

Electronic supplementary material The online version of this article (doi:10.1007/s12640-017-9720-2) contains supplementary material, which is available to authorized users.

Tatyana Strekalova

t.strekalova@maastrichtuniversity.nl

1 Section of Molecular Psychiatry, Clinical Research Unit of Disorders of Neurodevelopment and Cognition, Centre of Mental Health, University Hospital of Würzburg, University of Würzburg, Margarete-Höppel-Platz 1, 97080 Würzburg, Germany

2 Laboratory of Neurobiology of the Brain Integrative Functions, I.P. Pavlov Department of Physiology, Institute of Experimental Medicine, Akademika Pavlova 12, 197376 St. Petersburg, Russia

3 Laboratory of Biomolecular Screening, Institute of Physiologically Active Compounds, Russian Academy of Sciences, Severnii proezd 1, 142432 Chernogolovka, Moscow Region, Russia developmental brain plasticity factors MMP-9 and TIMP-1 were investigated in the medial prefrontal cortex and ventral/ dorsal hippocampus. LPS-challenged rats displayed moderately deficient escape responses in both memory tests, increased freezing behaviour and, surprisingly, reduced blood cortisol levels. Mmp9 and Timp1, and their ratio to one another, were differentially altered in pups versus adult untrained rats but remained unchanged overall in rats trained in either learning task. Together, our data indicate that systemic proinflammatory response during early postnatal development has long-lasting effects, including on the acquisition of motor escape abilities and plasticity factor expression, into adulthood. Our data suggest that altered stress response could possibly mediate these deviations and repeated training might generate positive effects on plasticity under the employed conditions.

4 Department of Neuroscience, Maastricht University, Universiteitssingel 40, NL, 6229 ER Maastricht, Netherlands

5 Laboratory of Molecular Mechanisms of Neuronal Interactions, I.M. Sechenov Institute of Evolutionary Physiology and Biochemistry, Russian Academy of Sciences, Thorez Avenue 44, 199223 St. Petersburg, Russia

6 Institute of Molecular Medicine, Laboratory of Psychiatric Neurobiology, I.M. Sechenov First Moscow State Medical University, Trubetskaya 8-2, 119991 Moscow, Russia

Department of Normal Physiology, I.M. Sechenov First Moscow State Medical University, Mokhovaya 11-4, 125009 Moscow, Russia 
Keywords Lipopolysaccharide (LPS) • MMP-9 · TIMP-1 · Escape learning $\cdot$ Corticosterone $\cdot$ RatAlexander Trofimov and Tatyana Strekalova equally contributed to this work.

\section{Introduction}

Inflammation during the early postnatal period has been implicated in the aetiology of numerous neuropsychiatric conditions including Alzheimer's disease, schizophrenia, attentiondeficit/hyperactivity disorder and autism (Rantakallio et al. 1997; Hornig et al. 1999; Shi et al. 2003; Bilbo and Schwarz 2012). Clinical and pre-clinical studies suggest that compromised brain neural plasticity is a pivotal pathophysiological link to these disorders. Early-life infection can increase levels of cytokines, such as interleukin (IL)-1 $\beta$, IL- 6 and tumour necrosis factor (TNF), and lead to impairments in attention and memory during adolescence and adulthood (de Bont et al. 1993; Aly et al. 2009; Yirmiya and Goshen 2011; Tishkina et al. 2016).

Postnatal administration of lipopolysaccharide (LPS) is a well-established model of the cognitive and behavioural effects of early-life systemic inflammation. Low-dose postnatal LPS administration increases cytokine production in a dosedependent manner and impairs memory in adulthood (Leonard 2001; Goshen et al. 2007; Donzis and Tronson 2014; Tishkina et al. 2016). The vast majority of studies have focused on evolutionarily late forms of memory, such as explicit learning, a hippocampus-dependent form of memory, which is known to be particularly vulnerable under various pathological conditions. Systemic LPS injection inhibits hippocampal long-term potentiation (LTP) (Vereker et al. 2000) and selectively impairs hippocampus-dependent spatial navigation in the Morris water maze and contextual fear conditioning, whereas cortex-independent auditory-cue fear conditioning remains unaffected (Rachal Pugh et al. 2001; Shaw et al. 2001). Little is known about the effects of early-life inflammation on the development of motor escape abilities, which are generally more preserved under pathological conditions.

Postnatal LPS administration attenuates plasticity-associated factors in the hippocampus and cortex including brain-derived neurotrophic factor (BDNF), nerve growth factor (NGF), neurotrophin-3 (NT-3) and $\mathrm{Ca}^{2+} /$ calmodulin-dependent protein kinase II (CaMKII), as well as altering TrkA, extracellular signal-regulated kinases and the expression of NMDA receptor subunit NR1 (Lapchak et al. 1993; Raetz and Whitfield 2002; Guan and Fang 2006; Schnydrig et al. 2007; Hennigan et al. 2007; Harré et al. 2008, Calabrese et al. 2014; Dehkordi et al. 2015). Meanwhile, striking differences between early stage and adult molecular and functional organization of the hippocampus question the relevance of these molecular mechanisms, as these adult plasticity markers are poorly expressed during earlylife (Travaglia et al. 2016). These concerns are supported by numerous in vitro findings showing opposing, stimulatory effects of pro-inflammatory cytokines on plasticity molecules, such as CaMKII, tyrosine kinases, mitogen-activated protein kinases (MAPKs), protein kinase $\mathrm{C}$ (PKC), phosphoinositide3 kinase (PI3K) and transcription factors such as nuclear factor kappa B (NF-KB) and activator protein 1 (AP-1) (Rosenberg 2002; Wu et al. 2004, 2009).

In the present work, we investigated messenger RNA (mRNA) levels of two functionally related developmental plasticity factors, tissue inhibitor of metalloproteinase 1 (TIMP-1) and matrix metalloproteinase 9 (MMP-9), after postnatal immune challenge with LPS. These factors help regulate neuronal remodelling and cell-to-cell interactions and are abundantly expressed in the prefrontal cortex and hippocampus (Ethell and Ethell 2007; Janusz et al. 2013; Aujla and Huntley 2014). MMP-9 is expressed in many cell types, including neurons and glia (Reinhard et al. 2015), and multiple brain regions including the prefrontal cortex and hippocampus (Bednarek et al. 2009; Aujla and Huntley 2014). MMP-9 is highly expressed during early brain development and decreases in adulthood (Aujla and Huntley 2014). A major function of MMP-9 is the regulation of cell-to-cell interactions by modifying the extracellular matrix (ECM), cell adhesion molecules, cell surface receptors, cytokines, growth factors and other proteases (Ethell and Ethell 2007; Vafadari et al. 2016). While MMP-9 levels are lower in the adult brain, its activity has been shown to increase in response to synaptic activity (Gawlak et al. 2009; Janusz et al. 2013).

One of the main mechanisms of MMP-9 activity regulation is via TIMP-1 which is secreted in response to synaptic activity at levels similar to MMP-9 (Ethell and Ethell 2007; Vafadari et al. 2016). While both their expression levels are low during adulthood, they remain functionally relevant as evidenced by the association of the compromised TIMP-1/ MMP-9 ratio to various CNS pathologies, including epilepsy, multiple sclerosis and bipolar disorder (Jourquin et al. 2005; Rybakowski et al. 2009; Reinhard et al. 2015). Proinflammatory cytokines have been repeatedly linked to abnormal expression of these genes (Jourquin et al. 2005; Okulski et al. 2007; Rybakowski et al. 2009; Berretta 2012).

Our previous studies suggest the P15-P21 rat postnatal development period to be sensitive to the administration of IL-1 $\beta$ (Zubareva et al. 2006, 2013; Trofimov et al. 2014, 2016). In a rat, the myelination and synaptogenesis in the prefrontal cortex and hippocampus peaks during this developmental period (O'Callaghan and Miller 1989; Rice and Barone 2000). In the present study, we investigate whether or not an early-life (P15-P21) pro-inflammatory LPS challenge that closely mimics clinical conditions alters motor learning in rats. As altered hypothalamic-pituitary-adrenal (HPA) axis functions are one of the long-lasting consequences of early-life inflammatory challenge and are known to negatively affect motor learning (Girard-Joyal et al. 2015; 
Kasahara et al. 2015), we also assessed hormonal and behavioural measures of stress response in postnatally challenged adult rats.

\section{Material and methods}

\section{Animals}

Two-month-old Wistar rats were obtained from a licensed provider, Rappolovo (Leningrad Region, Russia; licensed GOST-R-989112). Animals were housed under standard conditions (see Supporting Information). All studies conformed to the regulations outlined in the European Communities Council Directive (86/609/European Economic Community) and were approved by the ethics authorities of the Institute of Experimental Medicine, St. Petersburg.

\section{Study Outline}

One male and four female rats were co-housed until the females were pregnant, as described elsewhere (Pawluski et al. 2012); thereafter, females were single-housed until the pups were born. Experimental groups were balanced by body weight and received three injections of saline or LPS $(25 \mu \mathrm{g} /$ $\mathrm{kg}$ ) on days P15, P18, and P21 (Fig. 1a-f). In experiments I and II, LPS-challenged animals were sacrificed $2 \mathrm{~h}$ postinjection or on P81. Mmp9 and Timp1 were evaluated in the medial pre-frontal cortex (mPFC), dorsal hippocampus (DH) and ventral hippocampus (VH) using RT-PCR. In experiments III and IV, LPS-challenged rats were trained in a 5-day active avoidance footshock or a 4-day water maze task; $2 \mathrm{~h}$ after the last session, rats were killed and the above-indicated brain regions dissected for RT-PCR of Mmp9 and Timp1 mRNA. For experiments V and VI, LPS-challenged animals' openfield behaviour or serum was studied; an additional dose of $50 \mu \mathrm{g} / \mathrm{kg}$ of LPS was used in the latter study. Group sizes are indicated in Fig. 1. For details regarding LPS administration, see Supporting Information.

\section{Behavioural Tests}

\section{Active Avoidance Task}

During five consecutive days, rats were trained to associate light stimulation with a mild footshock. On day 1, they were placed in a customized transparent Plexiglas two-chamber shuttle box $(40 \times 30 \times 55 \mathrm{~cm})$ with grid floor (bars $1 \mathrm{~cm}$ apart) and after a 5-min acclimatization period were exposed to a 5-s light stimulation (lighting intensity on grid $110 \mathrm{~lx}$, lamp $10 \mathrm{~cm}$ above the grid) followed by a footshock (constant current of $0.5 \mathrm{~mA}, 1 \mathrm{~s}$ ) for ten consecutive sessions. During days $2-5$, each rat underwent 20 training sessions with random intersession intervals of 20-40 s. The percentage and latency of avoidance responses, defined as rat movement to a shockfree chamber after the conditioned stimulus but prior to shock, were recorded. Escape behaviour as defined by the displacement of a rat to a shock-free chamber after the shock delivery was scored as well.

Water Maze

Various A4 sheet size visual cues were placed on the inner walls of the tank $(\varnothing=150 \mathrm{~cm}$, walls $70 \mathrm{~cm}$ high) at positions north-east (NE), south-east (SE), south-west (SW) and northwest (NW). Four times a day on four consecutive days, animals were placed for $90 \mathrm{~s}$ in a pool of water mixed with milk, with 90 -s inter-trial intervals. A platform $(10 \times 10 \mathrm{~cm})$ was submerged $1 \mathrm{~cm}$ below water, at the centre of the NW sector (Trofimov et al. 2014). The four above-indicated starting points were randomly used as starting points for animals. After a rat reached the platform or was placed there when the trial elapsed, it was left there for $30 \mathrm{~s}$. Previous studies revealed no significant changes in spatial learning but instrumental-like performance under these conditions (Umriukhin and Strekalova, unpublished data). The percentage of animals that reached the platform within $90 \mathrm{~s}$ (escape response) and the average swimming speed were evaluated using the previously validated video-tracking program "Pavlovian Tracking” (St. Petersburg, Russia).

\section{Open Field}

Animals were placed at the centre of a black arena $(\varnothing=100 \mathrm{~cm}$, lighting intensity $5 \mathrm{~lx})$ for $3 \mathrm{~min}$ (Veniaminova and Zubareva 2015). The number of rearings and freezing events, defined by the absence of movements besides breathing, were recorded as described elsewhere (Strekalova et al. 2003; Vignisse et al. 2014). Behaviours were scored using the previously validated tracking program "Field4W" (St. Petersburg, Russia).

\section{Brain Dissection and qRT-PCR}

Brain dissection was carried out as described elsewhere (Morozova et al. 2016), and samples were immediately frozen and stored at $-70{ }^{\circ} \mathrm{C}$ until use. Total RNA was isolated using TRI Reagent (Molecular Research Center Inc., Cincinnati, $\mathrm{OH}$, USA). Two micrograms of total RNA was reversetranscribed into cDNA by M-MLV reverse transcriptase (Promega Corporation, Madison, WI, USA). Gene expression TaqMan assays were performed for Timp1, Mmp 9 and housekeeping gene Gapdh on the CFX96 Touch $^{\mathrm{TM}}$ Real-Time PCR Detection System (Bio-Rad Hercules, CA, USA). Glyceraldehyde 3-phosphate dehydrogenase (Gapdh) was chosen as a reference gene based on our previous results 
Fig. 1 Schematic outline of studies. In each study, rat pups received LPS injections on P15, $\mathrm{P} 18$ and P21. Expression of developmental plasticity factors was studied a $2 \mathrm{~h}$ post-injection (experiment I) and $\mathbf{b}$ in adulthood (experiment II). The following assays were performed: c footshock active avoidance (experiment III), d water maze escape learning (experiment IV), e open-field behaviour and $\mathbf{f}$ ELISA of plasma corticosterone
A

Experiment I

P15-P21

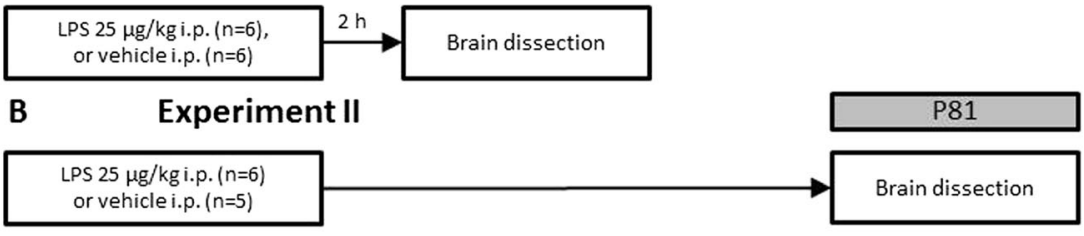

C Experiment III

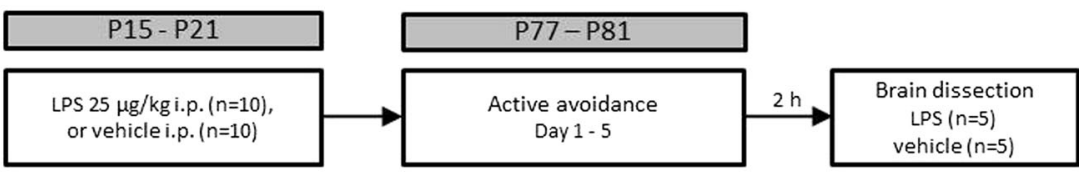

D Experiment IV

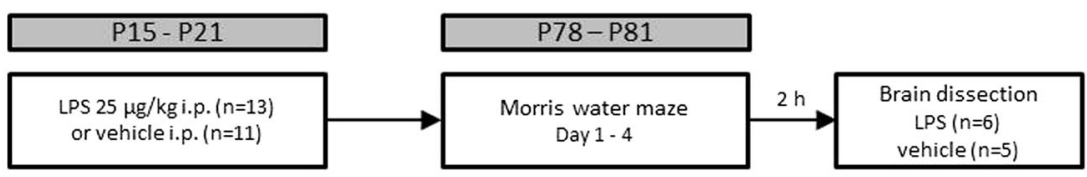

\section{E Experiment V}

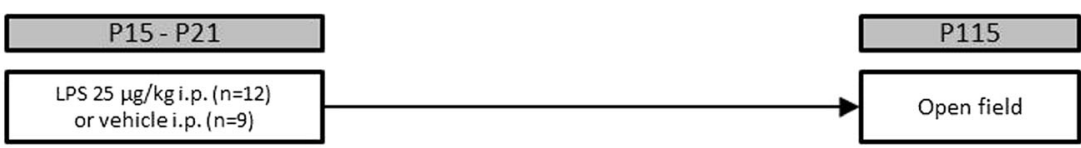

F

Experiment VI

P15-P21 corticosterone
(Couch et al. 2016). For primers and probe sequences (Alcor Bio, St. Petersburg, Russia) and cycling conditions, see Supporting Information. Relative mRNA levels were determined using the cycle threshold $(\mathrm{Ct})$ and the $2^{-\Delta \Delta \mathrm{Ct}}$ method as described previously (Couch et al. 2016) for both genes of interest, and the Timp 1:Mmp 9 ratio, a marker of developmental plasticity, was calculated (means of groups are in Table 2 of Supporting Information). All data were normalized to the means of respective control groups and expressed as a percentage.

\section{ELISA of Corticosterone}

Blood was collected, and samples of volume 100-200 $\mu$ l were left at room temperature for $60 \mathrm{~min}$ and then centrifuged at $800 \times \mathrm{g}$ for $10 \mathrm{~min}$ at room temperature. Serum was collected and analysed for corticosterone concentration with a DRG Corticosterone ELISA Kit (DRG International Inc., East Mountainside, MG, USA) in accordance with instructions of the manufacturer on a Microplate Reader Immunochem-2100 (HTI Diagnostics, Walpole, MA, USA).

\section{Statistical Analysis}

Data were analysed in Prism6.0 (GraphPad Software, Inc., USA). Two-group comparisons of gene expression and learning assays were performed using Mann-Whitney $U$ test, as these data did not pass the Shapiro-Wilk test for normal distribution. A three-group comparison of the normally distributed data from the ELISA study was carried out using ANOVA and Tukey's post hoc test. Linear regression was used to perform correlation analysis. Statistical significance was set at $p<0.05$. Data are shown as mean $\pm \mathrm{SEM}$.

\section{Results}

\section{Postnatal Administration of LPS Induces Differential Changes in Timp1 and Mmp9 in Pups versus Adult Rats and the Study of Escape Task Training in Adulthood}

In the mPFC, LPS-challenged pups had a significantly higher Timp 1:Mmp 9 ratio whereas naive LPS-challenged rats showed 
a tendency towards a reduced ratio compared with the vehicletreated control pups $(U=0.0, p=0.016$, Mann-Whitney $U$ test; $U=0.0, p=0.095$; respectively; Fig. $2 \mathrm{a}$; all means of relative fold expression data are presented in Supporting Information, Table 2). In comparison to control rats, Timp1 levels were significantly increased in LPS-challenged pups ( $U=0.0, p=0.01$; Fig. $2 \mathrm{~b}$ ) and significantly reduced in LPSchallenged adult rats $(U=2.0, p=0.032$; Fig. 2b). Adult LPSchallenged rats subjected to active avoidance or water maze training had a non-significant decrease of both Timp1:Mmp9 ratios and Timpl levels relative to controls $(U=8.5, p=0.46$ and $U=7.0, p=0.556$; respectively; Fig. 2b). None of the LPSchallenged groups displayed altered $\mathrm{Mmp} 9$ levels in comparison to control rats, while a non-significant optical decrease in expression levels was seen in the naïve LPS-treated rats (pups: $U=11.0, p=0.528$; untrained adults: $U=8.0, p=0.389$; adult active avoidance: $U=11.0, p=0.802$; adult water maze: $U=10.0, p=0.635$; Fig. 2c).

As for the DH, LPS-challenged pups had significantly higher Timp 1:Mmp9 ratios compared to controls while LPS-challenged untrained adults showed reduced ratios $(U=2.0, p=0.017$; $U=0.0, p=0.008$; respectively; Fig. $2 \mathrm{~d}$ ). Active avoidance or water maze paradigm-trained rats showed no ratio changes compared with controls $(U=7.0, p=0.310 ; U=7.0, p=0.310$; respectively; Fig. 2d); the latter group displayed a non-significant decrease of this measure. LPS-challenged pups had significantly increased Timpl in the $\mathrm{DH}(U=0.0, p=0.004)$ with a contrasting non-significant reduction of Timpl in adult LPS-challenged untrained rats and rats trained in active avoidance learning ( $U=5.0, p=0.171$ and $U=5.0, p=0.151$; respectively; Fig. 2e). No changes were found in LPS-challenged adult rats exposed to the water maze task ( $U=6.0, p=0.686$; Fig. 2 e). No significant changes in the Mmp 9 levels were found in any LPSchallenged rat groups in comparison to their respective vehicletreated controls (pups: $U=7.0, p=0.310$; adults: $U=8.0$, $p=0.397$; adult active avoidance: $U=9.0, p=0.532$; adult water maze: $U=5.0, p=0.486$; Fig. 2f).

Finally in the VH, LPS-challenged pups showed a significant increase of $\operatorname{Mmp} 9$ ( $U=1.0, p=0.019$; Fig. 2i) with a nonsignificant reduction in the Timp 1:Mmp 9 ratio $(U=8.0$, $p=0.474$; Fig. $2 \mathrm{~g}$ ) and a non-significant elevation of Timp1 ( $U=8.0, p=0.448$; Fig. $2 \mathrm{~h}$ ) relative to the respective control group. In LPS-challenged adult rats, no differences from controls were found in the Timp 1:Mmp9 ratio in the VH (untrained adults: $U=11.0, p=0.895$; adult active avoidance: $U=10.0, p=0.667$; adult water maze, $U=3.0, p=0.109$; Fig. $2 \mathrm{~g}$ ). Between these groups, no significant differences were found in Timpl in the VH (untrained adults: $U=12.0, p=1.000$; adult active avoidance: $U=11.0, p=0.847$; adult water maze, $U=4.0, p=0.168$; Fig. 2h). Similarly, no significant differences were found in Mmp9 in the VH (untrained adults: $U=11.0, p=0.914$; adult active avoidance: $U=10.0, p=0.690$; adult water maze, $U=3.0$, $p=0.111$; Fig. 2i). Thus, LPS administration in the early postnatal period resulted in aberrations of the TIMP1/MMP9 regulatory system across several regions of the developing limbic system, with directionally opposite changes in adulthood, which were not that evident in rats subjected to active avoidance and water maze.

\section{Postnatal Administration of LPS Results in Deficient Acquisition of Avoidance Task in the Adulthood}

On day 5, LPS-challenged rats showed significantly longer latency and a reduced percentage of avoidance responses than controls $(U=23.0, p=0.043 ; U=23.5, p=0.042$, respectively; Fig. 3a, b). The latency and percentage of avoidance responses on days $1-4$ of the active avoidance model were not significantly different between LPS-challenged and control animals (day 1: $U=47.0, p=0.836 ; U=47.5, p=0.777$, respectively; Fig. 3b; for days 2-4, see Supporting Information, Figs. 1 and 2). During all trials conducted in the experiment, either avoidance or escape behaviours were displayed by every rat, suggesting similar motor abilities and motivation across LPS- and non-LPS-challenged groups. As such, reduced avoidance memory as found in the LPS-treated animals is not likely to be due to an impairment other than in associative learning.

LPS-challenged rats had a smaller percentage of escape responses on day 1 of the water maze in comparison with controls $(U=38.5, p=0.049$; Fig. 3c). No differences were observed on day 4 ( $U=66.0, p=0.999$, respectively; Fig. 3c) and days 2-3 (see Supporting Information, Fig. 3). The swimming speed of LPS-exposed rats did not differ from controls (Fig. 3d). Also, we found that the mean escape latencies were not significantly different between the groups at any time point during the experiment ( $p>0.05$; Fig. 3e). Both vehicle- and LPS-challenged groups showed a significant correlation between the mean escape latency and the mean speed of swimming $(r=0.29, p=0.02$ and $r=0.58, p=0.001$, respectively; Fig. 3f). These data suggest a mild deficiency in motor tasks in LPS-challenged rats and rule out the possibility that other general factors not related to learning abilities impact the acquisition of escape responses in this assay.

\section{Altered Freezing Behaviour and Basal Plasma Corticosterone Levels in Adult Rats Subjected to Postnatal Administration of LPS}

In the open-field test, in comparison to control animals, LPSchallenged rats had more freezing events $(U=25.5, p=0.032)$ and similar number of rearings as controls $(U=38.0, p=0.272$; Fig. 4b), suggesting that the measures in locomotor activity are not related to the above-described group differences in learning scores. Postnatal challenge with LPS at a dose of 25 or $50 \mu \mathrm{g} / \mathrm{kg}$ resulted in decreased basal level of plasma corticosterone in comparison with controls; no significant difference was found between the two LPS dosage groups $\left(F_{(2,18)}=4.997, p=0.019\right.$, 


\section{Medial Prefrontal Cortex}

A Timp1/Mmp9 ratio

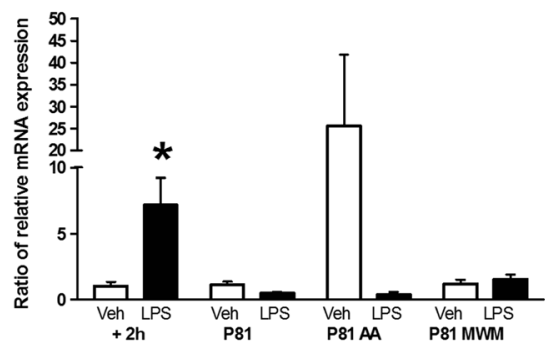

D Timp1/Mmp9 ratio

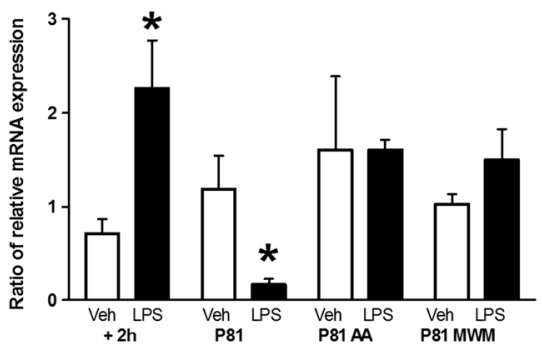

G Timp1/Mmp9 ratio

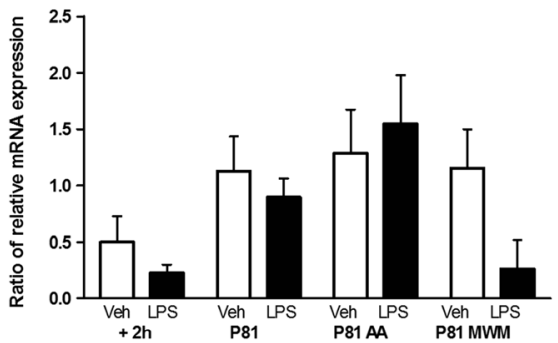

Fig. 2 Postnatal LPS administration differentially affects brain expression of Timpl and Mmp9 in pups and adult untrained rats. All comparisons are normalized to vehicle-treated controls. In the mPFC, a the LPS-challenged pup Timp 1:Mmp9 ratio was significantly higher, while adult LPS-treated, untrained rats had a tendency to a decreased ratio. b Timpl levels were significantly elevated in LPS-treated pups and decreased in LPS-challenged adult rats, and $\mathbf{c}$ there were no significant changes in Mmp9 in LPS-treated rats; adult LPS-treated rats trained in active avoidance or water maze tests had unaltered gene expression. In the DH, d LPS-treated pups showed significantly higher Timp 1:Mmp9

ANOVA; LPS 25 and $50 \mu \mathrm{g} / \mathrm{kg}: q=3.666, p=0.04 ; q=4.049$, $p=0.027$ versus control; $q=0.383, p=0.960$, respectively; Tukey test, Fig. 4c).

\section{Discussion}

In this study, we found persisting effects of postnatal systemic inflammatory challenge, on escape learning in the footshock-

\section{Mmp9 mRNA}

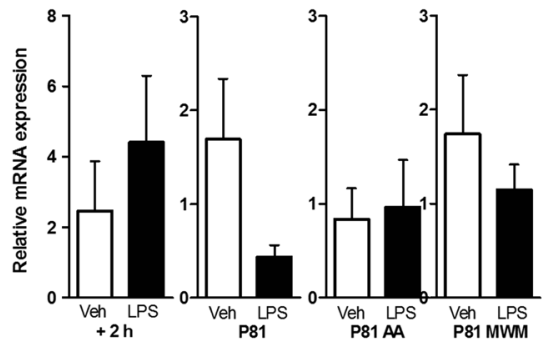

F Mmp9 mRNA

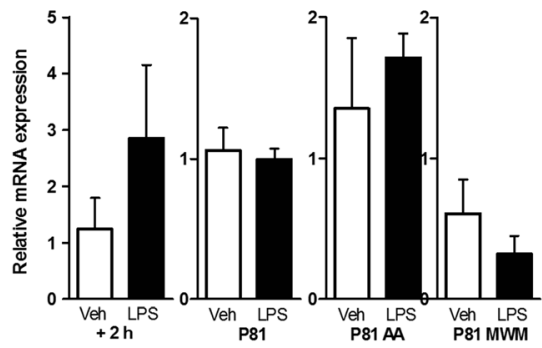

I Mmp9 mRNA

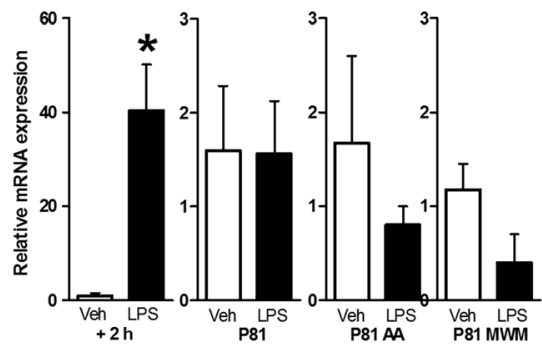

ratio, whereas adult untrained rats had opposite changes. e Timp1 was significantly elevated in LPS-treated pups, while adult rats showed a tendency to reduced Timp1. f No significant changes were found in Mmp9 in the LPS-challenged groups; rats subjected to training showed unaltered gene expression. In the VH, LPS-treated pups had a nonsignificant decrease of $\mathbf{g}$ Timp 1:Mmp 9 ratio, $\mathbf{h}$ a non-significant increase of Timpl and $\mathbf{i}$ a significant increase of Mmp 9 levels. $* p<0.05$ versus control. Veh vehicle-treated controls; LPS LPS-challenged; $A A$ active avoidance-trained rats; $W M$ water maze-trained rats. See the text

elicited active avoidance and water maze paradigms and on the expression of developmental plasticity factors TIMP-1/ $M M P-9$ in brain regions regulating emotionality and memory. These data suggest that early-life inflammation affects evolutionally ancient forms of learning, such as the acquisition of motor escape abilities, which are generally known to be robust under various pathological conditions. In our study, early life immune challenge of rats with LPS resulted in increased freezing behaviour and unexpectedly diminished 


\section{Active avoidance task}

A

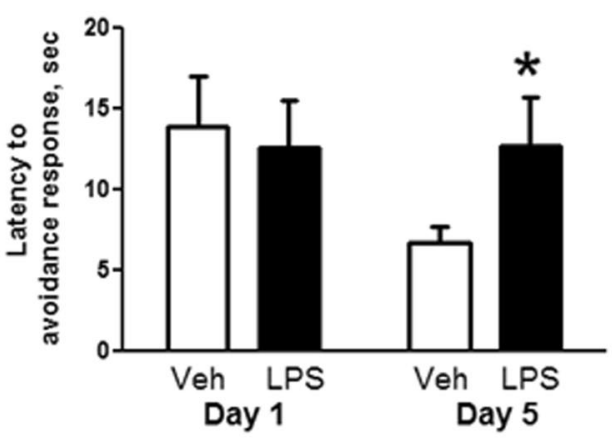

B

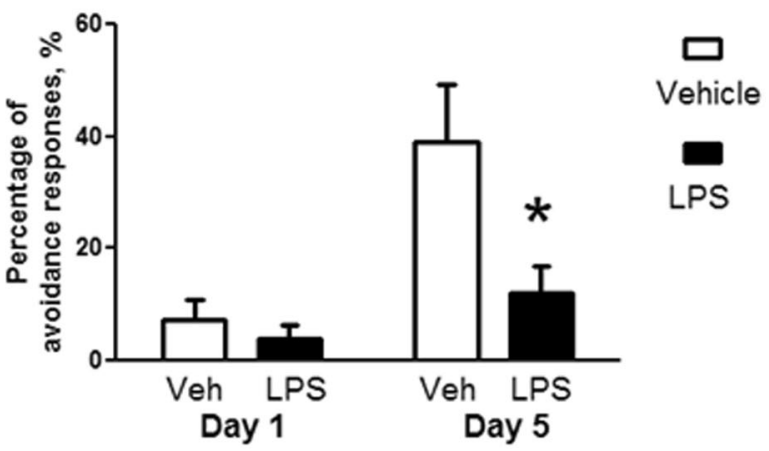

\section{Water maze}

C

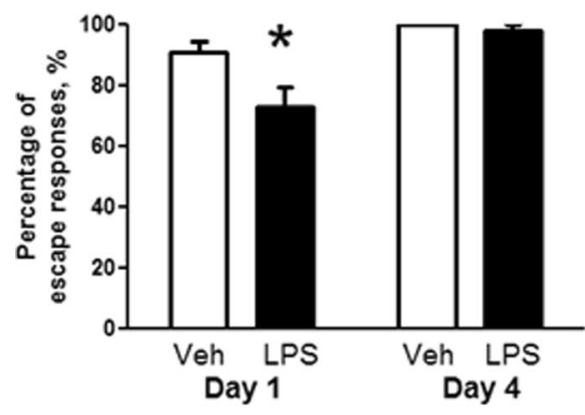

E

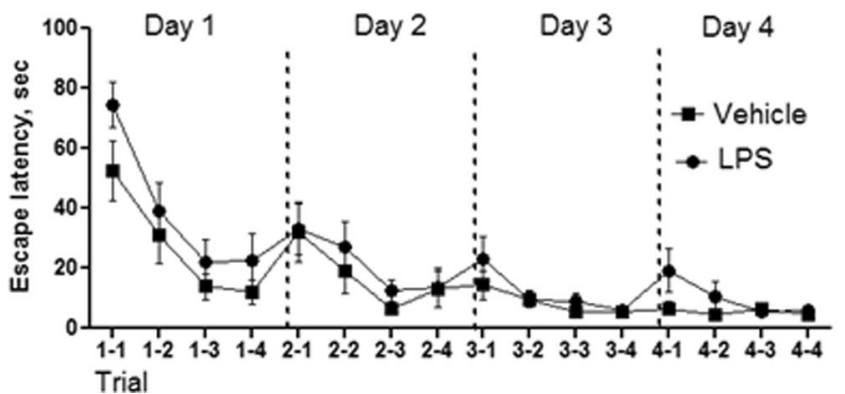

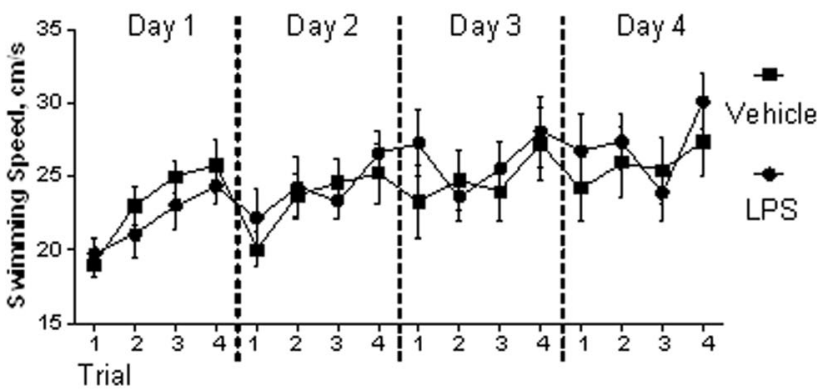

$\mathbf{F}$
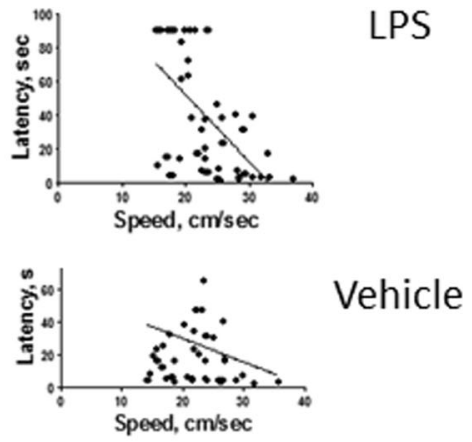

Fig. 3 Postnatal LPS treatment affects the acquisition of escape abilities in two memory paradigms. In the active avoidance task, LPS-challenged rats showed a longer latencies of avoidance response and $\mathbf{b}$ smaller percentage of avoidance responses than vehicle-treated rats, on day 5 but not day 1 . In the water maze, in comparison with controls, LPS-treated rats showed $\mathbf{c}$ a decreased percentage of avoidance responses on day 1 but not day 4 of training. LPS- and vehicle-injected animals showed similar d swimming speed and e mean escape latency. f In both LPS- and vehicle-treated rats, there was a significant correlation between swimming speed and escape latency. $* p<0.05$ versus controls. Abbreviations are as in Fig. 2 serum corticosterone levels in adulthood, suggesting altered mechanisms of stress response. A lack of significant molecular aberrations found in animals subjected to both postnatal LPS challenge and repeated escape training could be of potential functional importance.
Here, we report an increase in brain Timp1 and unchanged Mmp9 expression in LPS-challenged pups, which may shift the balance between these factors and impact MMP-9, an important regulator of morphological and synaptic plasticity (Bozdagi et al. 2007; Wlodarczyk et al. 2011). MMP-9 has 
Fig. 4 Postnatal LPS administration alters stress-related features. In the open-field test, as compared to controls, LPS-treated adult rats showed a a significantly elevated number of freezing events and $\mathbf{b}$ no changes in rearing activity. c Adult LPS-treated rats had significantly decreased basal levels of serum corticosterone in comparison with control rats $* p<0.05$ versus control animals. Abbreviations are as in Fig. 2

\section{Open field}

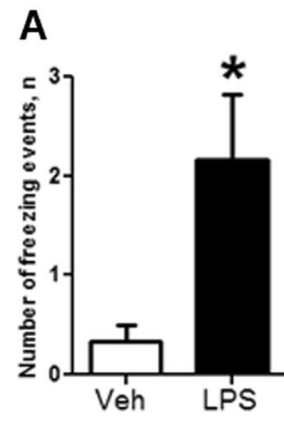

Serum corticosterone

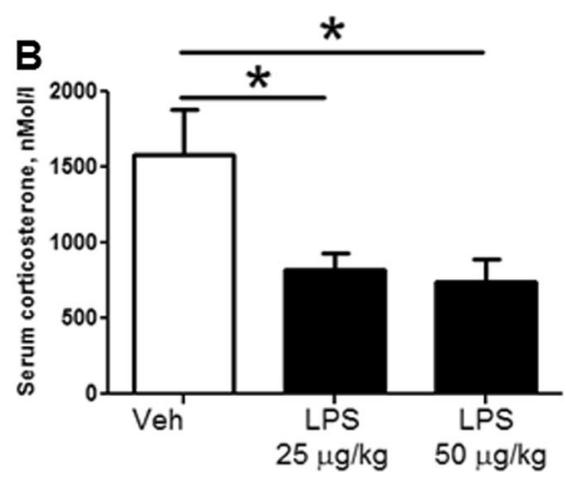

been shown to be involved in the modification of dendritic spines during neuronal stimulation and dendrite growth (Wang et al. 2008; Bilousova et al. 2009) possibly via regulation of the degradation of intercellular adhesion molecule (ICAM)-5 (Tian et al. 2007) and integrin- 31 -mediated signalling (Michaluk et al. 2011) and by increasing the lateral mobility of the NMDA receptors (Michaluk et al. 2011). In particular, incubation with MMP-9 changes the morphology of dendritic spines from a "more mature" mushroom-like form to a "less mature" filopodia-like form in a culture of neurons (Bilousova et al. 2009). Both decreases in MMP-9 expression and increases in TIMP-1 expression are related to the inhibition of LTP in the hippocampus. The development of the late phase of LTP was associated with increases of the concentration and proteolytic activity of MMP-9, while the inactivation of MMP-9 impaired LTP in the CA3-CA1 regions of hippocampal slices (Nagy et al. 2006). In vivo experiments have confirmed the role of MMP-9 in LTP induction and maintenance (Bozdagi et al. 2007). At the same time, TIMP-1 overexpression was shown to impair hippocampal LTP (Okulski et al. 2007). Given that synaptic remodelling and LTP induction are well-established parallels of learning abilities in rodent models, a dysregulation of TIMP-1/MMP-9 expression could underpin aberrant cognitive scores of LPS-challenged rats in our study.

The directions of molecular alterations induced by postnatal LPS injections were opposing in pups and adults, suggesting that potential compensatory processes occur in the TIMP1/MMP-9 system in adulthood. The occurrence of compensatory changes in the TIMP-1/MMP-9 pathway during adulthood supports its functional importance in the mature brain as previously suggested by clinical observations (Docherty et al. 1992; Bednarek et al. 2012). Two-wave changes in the expression of brain plasticity factors after postnatal inflammatory challenges have been reported previously: for example, LPS injection at P5 initially increased expression of the NMDA receptor NR1 subunit followed by a decrease in adulthood (Harré et al. 2008). Here, early postnatal challenge with LPS resulted in an increase in Timp1 expression which has been previously linked to reduced synaptic plasticity (Okulski et al. 2007). In contrast, adult animals which were postnatally injected with LPS showed a decrease in Timp1 expression and Timp1/Mmp9 ratio in the present study. Timp1 and Timp1/Mmp9 ratio reductions have been shown to improve synaptic plasticity, as discussed above (Nagy et al. 2006; Bozdagi et al. 2007). Since this group of rats displayed aberrant learning abilities, these deficits are likely to be due to alternative, possibly developmental, TIMP-1/ MMP-9-related mechanisms.

In the current study, molecular changes diverged between the investigated brain structures and seemed to be more pronounced in the mPFC and DH than in the VH. These differences are potentially a result of the different roles of these brain areas in mechanisms of learning versus stress response (Bagot et al. 2015) and the structure-specific effects of LPS that have been reported (Dinel et al. 2014). Previous studies demonstrated greater negative effects of LPS on neuronal survival and plasticity of dorsal versus ventral hippocampus (Järlestedt et al. 2013). Conversely, early-life stress predominantly affected plasticity of the ventral hippocampus and prefrontal cortex, but not dorsal hippocampus (Maras et al. 2014; Calabrese et al. 2015). Overly, all investigated brain areas have revealed expression changes in the TIMP-1/MMP-9 pathway which leads us to speculate that both mechanisms of plasticity and stress are affected in adult rats prenatally exposed to systemic inflammation.

Our study reveals short- and long-lasting effects of systemic inflammation on plasticity factors, which are particularly important during development and have so far been addressed in a very limited number of studies. This approach of identifying developmental molecules vulnerable to systemic inflammation rather than those known to be involved in adult brain plasticity could be of greater importance to furthering our understanding of the primary mechanisms through which 
early-life stress can lead to impairments in adult brain functions.

Postnatally LPS-challenged rats exposed to the active avoidance or water maze learning showed no significant changes in the expression of Timpl or Mmp9, while similar to the untrained LPS-treated group, a non-significant movement towards compromised Timp 1 and Timp $1 / M m p 9$ ratio in the prefrontal cortex was observed in rats subjected to active avoidance. LPS-challenged rats trained in the water maze task have shown an optical reduction of all three parameters in ventral hippocampus; however, the differences in expression levels in the untrained LPS-treated group were far from significant. A lack of significant changes in the investigated plasticity factors in repeatedly trained LPS-challenged rats could be interpreted as a consequence of normalizing synaptic remodelling which is a well-established effect of chronic training in various memory tasks (Pereira et al. 2007; Stamatakis et al. 2014; Smolen et al. 2016). At the same time, the large variability and low levels of Timp1 and Mmp 9 found in this study, as is characteristic of the expression of developmental factors during adulthood, limit the strength of this hypothesis.

Early-life inflammatory challenge was found in our work to result in deficient motor learning of active avoidance and water maze tasks. Up to now, the majority of literature regarding the effects of postnatal systemic inflammation on brain plasticity predominantly reported changes in hippocampusdependent learning. However, several observations are in line with our results and suggest that postnatal inflammation can affect other forms of memory as well. Postnatal LPS injection impairs object recognition memory (Hennigan et al. 2007), spontaneous alteration and working memory in the T maze (Hauss-Wegrzyniak et al. 1998), while high-dosage LPS disrupts instrumental learning of the instrumental flexion response (Young et al. 2007). Thus, early-life systemic inflammation affects not only evolutionarily novel types of memory, which are known to be particularly vulnerable under various pathological conditions (Vereker et al. 2000; Rachal Pugh et al. 2001; Shaw et al. 2001), but also more basic forms of learning, such as the acquisition of motor escape abilities, which are generally less disrupted by deleterious factors.

In the present study, LPS-challenged rats showed deficient learning at the beginning of the water maze training, while in the active avoidance paradigm, memory deficits occurred at the end of the experiment. Based on the above-discussed behavioural and gene expression assay results, it can be speculated that in repeatedly trained LPS-challenged rats, the dynamics of learning deficits parallel the altered expression of developmental plasticity genes along with other unknown functional changes. The different time points at which deficits of acquisition occur in the applied memory tests may be due to the different roles of the hippocampus and prefrontal cortex, investigated plasticity factors and HPA-related mechanisms, in the two rodent models of learning.
For example, previous studies have revealed a link between neophobia and deficits in motor learning and other forms of memory (Hernadi et al. 1997; Strekalova et al. 2013; Sarowar et al. 2016), including the water maze task ( $\mathrm{Li}$ et al. 2002; Kelly et al. 2003). While the factor of neophobia could be substantial during the first sessions of the water maze training of LPS-treated rats, it is unlikely to have played a significant role at later training sessions, nor during the active avoidance paradigm, where animals were exposed to pre-training habituation. In the latter paradigm, however, a stress impact of training might be of importance, since repeated footshock is well known to induce stress response in rodents particularly, affecting glucocorticoids (Rosecrans et al. 1986; D'Hooge and De Deyn 2001). Altered neophobic responses and blood corticosterone levels are known to involve HPA-related mechanisms, the change of which is one of the major features of early-life systemic inflammation (Shanks et al. 1995; Ellis et al. 2006; Dinel et al. 2014).

Evidence for altered HPA activity was found in the present work. Our study revealed increased freezing behaviour in postnatally LPS-challenged rats tested under mild lighting conditions in a novel open-field situation, where anxiety-like behaviours are normally not evoked in rodents (Strekalova et al. 2005; Strekalova and Steinbusch 2010). Previous studies that employed open-field testing with similar illumination strength have demonstrated behavioural abnormalities in mice and rats with altered HPA activity (Couch et al. 2013; Pawluski et al. 2012). Hence, our present findings suggest an increased HPA responsiveness to stress in postnatally LPS-challenged rats. Indeed, various aberrations in neuronal functions at adulthood associated with early-life systemic inflammation are considered to result from elevated HPA axis activity. Inflammatory activation of the HPA axis was shown to provide an important regulatory feedback to the proinflammatory cytokines that limits their synthesis (Besedovsky et al. 1986; Del Rey et al. 1987). In rats, exposure to LPS in early life increases corticotrophin-releasing hormone expression in the hypothalamus, decreasing glucocorticoid receptor density in the hypothalamus, hippocampus and frontal cortex (Shanks et al. 1995) and affecting its phosphorylation (Dinel et al. 2014).

While most previous studies carried out on adults report increased corticosterone levels after systemic inflammatory challenge (Kohman et al. 2008; Kasahara et al. 2015; Girard-Joyal et al. 2015), we found that early-life administration of two different doses of LPS produced a prominent decrease in corticosterone, suggesting compensatory changes in the HPA system. Various challenges that normally increase HPA activity and corticosterone levels in adulthood (Maccari et al. 2003; Weinstock 2008) have been shown to have no effect (Koenig et al. 2005; Weinstock 2008; Dinel et al. 2014) or, like in our study, result in decreases (Cannizzaro et al. 2006; Solati et al. 2015) when applied 
during development. One of the explanations for the paradoxical decrease of plasma corticosterone in our work can be due to the previously discussed over-expression of hypothalamic hormones regulating adrenal function after early-life LPS injection, which may be followed by a compensatory suppression of glucocorticoid production (Shanks et al. 1995; Dinel et al. 2014). The direction of corticosterone changes in rodents postnatally treated with LPS was shown to be linked to the development period (Dinel et al. 2014; Girard-Joyal et al. 2015). For instance, the effects of LPS on blood corticosterone levels in mice were significantly weaker at the peripubertal/ adolescent period, particularly in males.

Together, our data concerning increased freezing behaviour and decreased plasma corticosterone levels suggest that earlylife systemic inflammation generates long-lasting alternations in stress response and HPA axis functions. The long-lasting impact of an early-life immune challenge on HPA responsiveness is linked to its tight relationship with neuroimmune mechanisms (Spencer et al. 2006; Dinel et al. 2014). HPA axis activation in animals can be altered by early-life LPS activation which may result in abnormal triggering of the complex regulatory responses of stress hormones to environmental factors (Besedovsky et al. 1986; Mouihate et al. 2010). Such an abnormality in response could occur in response to the novelty stress which rats are exposed to during our open-field paradigm, which might evoke the paradoxical effect of a suppression of corticosterone release.

In summation, postnatal systemic inflammation compromises motor escape learning in adulthood, accompanied by aberrant expression of the TIMP-1/MMP-9 developmental plasticity pathway. These abnormalities, along with alterations in both hormonal and behavioural markers of stress response, suggest that the learning deficits associated with early-life LPS challenge may arise as a result of disruptions to the plasticity-associated TIMP-1/MMP-9 cascade and aberrant HPA activity.

Acknowledgements We acknowledge the help of Mrs. Ekaterina Veniaminova and Mr. Kevin Fomalont, as well as the financial support of RFBR16-34-00316, AGGRESSOTYPE FP7/No. 602805, DAAD to AT, and the "5-100" Russian Scientific Excellence program.

\section{Compliance with Ethical Standards}

Conflicts of Interest The authors declare that they have no conflict of interest.

Ethical Approval All experiments described followed the European Communities Council Directive (86/609/European Economic Community) and were approved by the ethics authorities of the Institute of Experimental Medicine, St. Petersburg.

Open Access This article is distributed under the terms of the Creative Commons Attribution 4.0 International License (http:// creativecommons.org/licenses/by/4.0/), which permits unrestricted use, distribution, and reproduction in any medium, provided you give appropriate credit to the original author(s) and the source, provide a link to the Creative Commons license, and indicate if changes were made.

\section{References}

Aly H, Abd-Rabboh L, El-Dib M et al (2009) Ascorbic acid combined with ibuprofen in hypoxic ischemic encephalopathy: a randomized controlled trial. J Perinatol 29:438-443

Aujla PK, Huntley GW (2014) Early postnatal expression and localization of matrix metalloproteinases-2 and -9 during establishment of rat hippocampal synaptic circuitry. J Comp Neurol 522:1249-1263

Bagot RC, Parise EM, Peña CJ et al (2015) Ventral hippocampal afferents to the nucleus accumbens regulate susceptibility to depression. Nat Commun 6:7062

Bednarek N, Clément Y, Lelièvre V et al (2009) Ontogeny of MMPs and TIMPs in the murine neocortex. Pediatr Res 65:296-300

Bednarek N, Svedin P, Garnotel R et al (2012) Increased MMP-9 and TIMP-1 in mouse neonatal brain and plasma and in human neonatal plasma after hypoxia-ischemia: a potential marker of neonatal encephalopathy. Pediatr Res 71:63-70

Berretta S (2012) Extracellular matrix abnormalities in schizophrenia. Neuropharmacology 62:1584-1597

Besedovsky H, del Rey A, Sorkin E, Dinarello CA (1986) Immunoregulatory feedback between interleukin-1 and glucocorticoid hormones. Science 233:652-654

Bilbo SD, Schwarz JM (2012) The immune system and developmental programming of brain and behavior. Front Neuroendocrinol 33: $267-286$

Bilousova TV, Dansie L, Ngo M et al (2009) Minocycline promotes dendritic spine maturation and improves behavioural performance in the fragile X mouse model. J Med Genet 46:94-102

Bozdagi O, Nagy V, Kwei KT, Huntley GW (2007) In vivo roles for matrix metalloproteinase-9 in mature hippocampal synaptic physiology and plasticity. J Neurophysiol 98:334-344

Calabrese F, Rossetti AC, Racagni G et al (2014) Brain-derived neurotrophic factor: a bridge between inflammation and neuroplasticity. Front Cell Neurosci 8:430

Calabrese F, van der Doelen RHA, Guidotti G et al (2015) Exposure to early life stress regulates Bdnf expression in SERT mutant rats in an anatomically selective fashion. J Neurochem 132:146-154

Cannizzaro C, Plescia F, Martire M et al (2006) Single, intense prenatal stress decreases emotionality and enhances learning performance in the adolescent rat offspring: interaction with a brief, daily maternal separation. Behav Brain Res 169:128-136

Couch Y, Anthony DC, Dolgov O et al (2013) Microglial activation, increased TNF and SERT expression in the prefrontal cortex define stress-altered behaviour in mice susceptible to anhedonia. Brain Behav Immun 29:136-146

Couch Y, Trofimov A, Markova N et al (2016) Low-dose lipopolysaccharide (LPS) inhibits aggressive and augments depressive behaviours in a chronic mild stress model in mice. J Neuroinflammation 13:108

D'Hooge R, De Deyn PP (2001) Applications of the Morris water maze in the study of learning and memory. Brain Res Brain Res Rev 36: 60-90

de Bont ES, Martens A, van Raan J et al (1993) Tumor necrosis factoralpha, interleukin-1 beta, and interleukin-6 plasma levels in neonatal sepsis. Pediatr Res 33:380-383

Dehkordi NG, Noorbakhshnia M, Ghaedi K et al (2015) Omega-3 fatty acids prevent LPS-induced passive avoidance learning and memory and CaMKII- $\alpha$ gene expression impairments in hippocampus of rat. Pharmacol Rep 67:370-375 
Del Rey A, Besedovsky H, Sorkin E, Dinarello CA (1987) Interleukin-1 and glucocorticoid hormones integrate an immunoregulatory feedback circuit. Ann N Y Acad Sci 496:85-90

Dinel A-L, Joffre C, Trifilieff P et al (2014) Inflammation early in life is a vulnerability factor for emotional behavior at adolescence and for lipopolysaccharide-induced spatial memory and neurogenesis alteration at adulthood. J Neuroinflammation 11:155

Docherty AJ, O'Connell J, Crabbe T et al (1992) The matrix metalloproteinases and their natural inhibitors: prospects for treating degenerative tissue diseases. Trends Biotechnol 10:200-207

Donzis EJ, Tronson NC (2014) Modulation of learning and memory by cytokines: signaling mechanisms and long term consequences. Neurobiol Learn Mem 115:68-77

Ellis S, Mouihate A, Pittman QJ (2006) Neonatal programming of the rat neuroimmune response: stimulus specific changes elicited by bacterial and viral mimetics. J Physiol 571:695-701

Ethell IM, Ethell DW (2007) Matrix metalloproteinases in brain development and remodeling: synaptic functions and targets. J Neurosci Res 85:2813-2823

Gawlak M, Górkiewicz T, Gorlewicz A et al (2009) High resolution in situ zymography reveals matrix metalloproteinase activity at glutamatergic synapses. Neuroscience 158:167-176

Girard-Joyal O, Faragher A, Bradley K et al (2015) Age and sex differences in c-Fos expression and serum corticosterone concentration following LPS treatment. Neuroscience 305:293-301

Goshen I, Kreisel T, Ounallah-Saad H et al (2007) A dual role for interleukin-1 in hippocampal-dependent memory processes. Psychoneuroendocrinology 32:1106-1115

Guan Z, Fang J (2006) Peripheral immune activation by lipopolysaccharide decreases neurotrophins in the cortex and hippocampus in rats. Brain Behav Immun 20:64-71

Harré E-M, Galic MA, Mouihate A et al (2008) Neonatal inflammation produces selective behavioural deficits and alters N-methyl-Daspartate receptor subunit mRNA in the adult rat brain. Eur J Neurosci 27:644-653

Hauss-Wegrzyniak B, Dobrzanski P, Stoehr JD, Wenk GL (1998) Chronic neuroinflammation in rats reproduces components of the neurobiology of Alzheimer's disease. Brain Res 780:294-303

Hennigan A, Trotter C, Kelly AM (2007) Lipopolysaccharide impairs long-term potentiation and recognition memory and increases p75NTR expression in the rat dentate gyrus. Brain Res 1130:158166

Hernadi I, Karadi Z, Faludi B, Lenard L (1997) Disturbances of neophobia and taste-aversion learning after bilateral kainate microlesions in the rat pallidum. Behav Neurosci 111:137-146

Hornig M, Weissenböck H, Horscroft N, Lipkin WI (1999) An infectionbased model of neurodevelopmental damage. Proc Natl Acad Sci U S A 96:12102-12107

Janusz A, Milek J, Perycz M et al (2013) The fragile X mental retardation protein regulates matrix metalloproteinase $9 \mathrm{mRNA}$ at synapses. J Neurosci 33:18234-18241

Järlestedt K, Naylor AS, Dean J et al (2013) Decreased survival of newborn neurons in the dorsal hippocampus after neonatal LPS exposure in mice. Neuroscience 253:21-28

Jourquin J, Tremblay E, Bernard A et al (2005) Tissue inhibitor of metalloproteinases-1 (TIMP-1) modulates neuronal death, axonal plasticity, and learning and memory. Eur J Neurosci 22:2569-2578

Kasahara E, Sekiyama A, Hori M et al (2015) Stress-induced glucocorticoid release upregulates uncoupling protein-2 expression and enhances resistance to endotoxin-induced lethality. Neuroimmunomodulation 22:279-292

Kelly A, Vereker E, Nolan Y et al (2003) Activation of p38 plays a pivotal role in the inhibitory effect of lipopolysaccharide and interleukin-1 beta on long term potentiation in rat dentate gyrus. J Biol Chem 278: 19453-19462
Koenig JI, Elmer GI, Shepard PD et al (2005) Prenatal exposure to a repeated variable stress paradigm elicits behavioral and neuroendocrinological changes in the adult offspring: potential relevance to schizophrenia. Behav Brain Res 156:251-261

Kohman RA, Tarr AJ, Sparkman NL et al (2008) Neonatal endotoxin exposure impairs avoidance learning and attenuates endotoxininduced sickness behavior and central IL-1beta gene transcription in adulthood. Behav Brain Res 194:25-31

Lapchak P a, Araujo DM, Hefti F (1993) Systemic interleukin-1 beta decreases brain-derived neurotrophic factor messenger RNA expression in the rat hippocampal formation. Neuroscience 53:297-301

Leonard BE (2001) Changes in the immune system in depression and dementia: causal or co-incidental effects? Int J Dev Neurosci 19: 305-312

Li XL, Aou S, Hori T, Oomura Y (2002) Spatial memory deficit and emotional abnormality in OLETF rats. Physiol Behav 75:15-23

Maccari S, Darnaudery M, Morley-Fletcher S et al (2003) Prenatal stress and long-term consequences: implications of glucocorticoid hormones. Neurosci Biobehav Rev 27:119-127

Maras PM, Molet J, Chen Y et al (2014) Preferential loss of dorsalhippocampus synapses underlies memory impairments provoked by short, multimodal stress. Mol Psychiatry 19:811-822

Michaluk P, Wawrzyniak M, Alot P et al (2011) Influence of matrix metalloproteinase MMP-9 on dendritic spine morphology. J Cell Sci 124:3369-3380

Morozova A, Zubkov E, Strekalova T et al (2016) Ultrasound of alternating frequencies and variable emotional impact evokes depressive syndrome in mice and rats. Prog Neuro-Psychopharmacol Biol Psychiatry 68:52-63

Mouihate A, Galic MA, Ellis SL et al (2010) Early life activation of tolllike receptor 4 reprograms neural anti-inflammatory pathways. J Neurosci 30:7975-7983

Nagy V, Bozdagi O, Matynia A et al (2006) Matrix metalloproteinase-9 is required for hippocampal late-phase long-term potentiation and memory. J Neurosci 26:1923-1934

O'Callaghan JP, Miller DB (1989) Assessment of chemically-induced alterations in brain development using assays of neuron- and glialocalized proteins. Neurotoxicology 10:393-406

Okulski P, Jay TM, Jaworski J et al (2007) TIMP-1 abolishes MMP-9dependent long-lasting long-term potentiation in the prefrontal cortex. Biol Psychiatry 62:359-362

Pawluski JL, Valença A, Santos AIM et al (2012) Pregnancy or stress decrease complexity of $\mathrm{CA} 3$ pyramidal neurons in the hippocampus of adult female rats. Neuroscience 227:201-210

Pereira AC, Huddleston DE, Brickman AM et al (2007) An in vivo correlate of exercise-induced neurogenesis in the adult dentate gyrus. Proc Natl Acad Sci U S A 104:5638-5643

Rachal Pugh C, Fleshner M, Watkins LR et al (2001) The immune system and memory consolidation: a role for the cytokine IL-1beta. Neurosci Biobehav Rev 25:29-41

Raetz CRH, Whitfield C (2002) Lipopolysaccharide endotoxins. Annu Rev Biochem 71:635-700

Rantakallio P, Jones P, Moring J, Von Wendt L (1997) Association between central nervous system infections during childhood and adult onset schizophrenia and other psychoses: a 28-year follow-up. Int J Epidemiol 26:837-843

Reinhard SM, Razak K, Ethell IM (2015) A delicate balance: role of MMP-9 in brain development and pathophysiology of neurodevelopmental disorders. Front Cell Neurosci 9:280

Rice D, Barone S (2000) Critical periods of vulnerability for the developing nervous system: evidence from humans and animal models. Environ Health Perspect 108(Suppl):511-533

Rosecrans JA, Robinson SE, Johnson JH et al (1986) Neuroendocrine, biogenic amine and behavioral responsiveness to a repeated footshock-induced analgesia (FSIA) stressor in Sprague-Dawley (CD) and Fischer-344 (CDF) rats. Brain Res 382:71-80 
Rosenberg GA (2002) Matrix metalloproteinases in neuroinflammation. Glia 39:279-291

Rybakowski JK, Skibinska M, Leszczynska-Rodziewicz A et al (2009) Matrix metalloproteinase-9 gene and bipolar mood disorder. NeuroMolecular Med 11:128-132

Sarowar T, Grabrucker S, Föhr K et al (2016) Enlarged dendritic spines and pronounced neophobia in mice lacking the PSD protein RICH2. Mol Brain 9:28

Schnydrig S, Korner L, Landweer S et al (2007) Peripheral lipopolysaccharide administration transiently affects expression of brainderived neurotrophic factor, corticotropin and proopiomelanocortin in mouse brain. Neurosci Lett 429:69-73

Shanks N, Larocque S, Meaney MJ (1995) Neonatal endotoxin exposure alters the development of the hypothalamic-pituitary-adrenal axis: early illness and later responsivity to stress. J Neurosci 15:376-384

Shaw KN, Commins S, O’Mara SM (2001) Lipopolysaccharide causes deficits in spatial learning in the watermaze but not in BDNF expression in the rat dentate gyrus. Behav Brain Res 124:47-54

Shi L, Fatemi SH, Sidwell RW, Patterson PH (2003) Maternal influenza infection causes marked behavioral and pharmacological changes in the offspring. J Neurosci 23:297-302

Smolen P, Zhang Y, Byrne JH (2016) The right time to learn: mechanisms and optimization of spaced learning. Nat Rev Neurosci 17:77-88

Solati J, Kleehaupt E, Kratz O et al (2015) Inverse effects of lipopolysaccharides on anxiety in pregnant mice and their offspring. Physiol Behav 139:369-374

Spencer SJ, Boissé L, Mouihate A, Pittman QJ (2006) Long term alterations in neuroimmune responses of female rats after neonatal exposure to lipopolysaccharide. Brain Behav Immun 20:325-330

Stamatakis A, Diamantopoulou A, Panagiotaropoulos T et al (2014) A novel model of early experiences involving neonatal learning of a Tmaze using maternal contact as a reward or its denial as an event of mild emotional adversity. Dev Psychobiol 56:1651-1660

Strekalova T, Steinbusch HWM (2010) Measuring behavior in mice with chronic stress depression paradigm. Prog Neuro-Psychopharmacol Biol Psychiatry 34:348-361

Strekalova T, Zörner B, Zacher C et al (2003) Memory retrieval after contextual fear conditioning induces c-Fos and JunB expression in CA1 hippocampus. Genes Brain Behav 2:3-10

Strekalova T, Spanagel R, Dolgov O, Bartsch D (2005) Stress-induced hyperlocomotion as a confounding factor in anxiety and depression models in mice. Behav Pharmacol 16:171-180

Strekalova T, Anthony DC, Dolgov O et al (2013) The differential effects of chronic imipramine or citalopram administration on physiological and behavioral outcomes in naïve mice. Behav Brain Res 245:101106

Tian L, Stefanidakis M, Ning L et al (2007) Activation of NMDA receptors promotes dendritic spine development through MMP-mediated ICAM-5 cleavage. J Cell Biol 178:687-700

Tishkina A, Stepanichev M, Kudryashova I et al (2016) Neonatal proinflammatory challenge in male Wistar rats: effects on behavior, synaptic plasticity, and adrenocortical stress response. Behav Brain Res 304:1-10
Travaglia A, Bisaz R, Cruz E, Alberini CM (2016) Developmental changes in plasticity, synaptic, glia and connectivity protein levels in rat dorsal hippocampus. Neurobiol Learn Mem 135:125-138

Trofimov AN, Zubareva OE, Simbirtsev AS, Klimenko VM (2014) Effects of neonatal increases in interleukin- $1 \beta$ levels on the formation of spatial memory in adult rats. Neurosci Behav Physiol 44: 359-364

Trofimov AN, Zubareva OE, Shvarts AP et al (2016) Expression of the FGF2 and TIMP1 genes in the adult rat brain after administration of interleukin-1beta during early postnatal ontogeny. Neurosci Behav Physiol 46:1-8

Vafadari B, Salamian A, Kaczmarek L (2016) MMP-9 in translation: from molecule to brain physiology, pathology, and therapy. J Neurochem S2:91-114

Veniaminova EA, Zubareva OE (2015) The changes in exploratory behavior and Fgf2 gene expression in cells of the rat brain after the early postnatal administration of bacterial lipopolysaccharide. Neurochem J 9:127-132

Vereker E, Campbell V, Roche E et al (2000) Lipopolysaccharide inhibits long term potentiation in the rat dentate gyrus by activating caspase1. J Biol Chem 275:26252-26258

Vignisse J, Steinbusch HWM, Grigoriev V et al (2014) Concomitant manipulation of murine NMDA- and AMPA-receptors to produce pro-cognitive drug effects in mice. Eur Neuropsychopharmacol 24: 309-320

Wang X, Bozdagi O, Nikitczuk JS et al (2008) Extracellular proteolysis by matrix metalloproteinase- 9 drives dendritic spine enlargement and long-term potentiation coordinately. Proc Natl Acad Sci U S A 105:19520-19525

Weinstock M (2008) The long-term behavioural consequences of prenatal stress. Neurosci Biobehav Rev 32:1073-1086

Wlodarczyk J, Mukhina I, Kaczmarek L, Dityatev A (2011) Extracellular matrix molecules, their receptors, and secreted proteases in synaptic plasticity. Dev Neurobiol 71:1040-1053

Wu C-Y, Hsieh H-L, Jou M-J, Yang C-M (2004) Involvement of p42/p44 MAPK, p38 MAPK, JNK and nuclear factor-kappa B in interleukin1 beta-induced matrix metalloproteinase- 9 expression in rat brain astrocytes. J Neurochem 90:1477-1488

Wu CY, Hsieh HL, Sun CC, Yang CM (2009) IL-1 $\beta$ induces MMP-9 expression via a $\mathrm{Ca} 2+-$ dependent $\mathrm{CaMKII/JNK/c-Jun} \mathrm{cascade} \mathrm{in}$ rat brain astrocytes. Glia 57:1775-1789

Yirmiya R, Goshen I (2011) Immune modulation of learning, memory, neural plasticity and neurogenesis. Brain Behav Immun 25:181-213

Young EE, Baumbauer KM, Elliot A, Joynes RL (2007) Lipopolysaccharide induces a spinal learning deficit that is blocked by IL-1 receptor antagonism. Brain Behav Immun 21:748-757

Zubareva OE, Eliseeva AP, Simbirtsev AS, Klimenko VM (2006) The effects of proinflammatory cytokines on the formation of behavior in early postnatal ontogenesis. Neurosci Behav Physiol 36:367-372

Zubareva OE, Shcherbakova KP, Kalemenev SV et al (2013) Impairments to conditioned reflex activity in adult rats after administration of interleukin- $1 \beta$ during early postnatal ontogeny. Neurosci Behav Physiol 43:210-213 